\title{
Comparison between simulation results and DEXA investigation of the bone remodelling after implanting a cementless long stem hip prosthesis
}

\author{
Almohallami A ${ }^{1}$, Bouguecha $\mathrm{A}^{1}$, Stukenborg-Colsman $\mathrm{C}^{2}$, Lerch $\mathrm{M}^{2}$, Nolte $\mathrm{I}^{3}$, Behrens B-A. ${ }^{1}$ \\ ${ }^{1}$ Institute of forming technology and machines, Leibniz Universität Hannover, Germany \\ ${ }^{2}$ Department of Orthopaedics (Annastift), Hannover medical school, Germany \\ ${ }^{3}$ Small Animal Clinic, University of veterinary medicine Hannover, foundation, Germany
}

almohallami@ifum.uni-hannover.de

\begin{abstract}
Bone remodelling around a femoral prosthesis is one of the main reasons of aseptic loosening of the implant's stem. The difference in stiffness between the bone tissue and the prosthesis leads to unload the periprosthetic bone and thus to decrease the bone mineral density (BMD), which is known as stress shielding. This paper represents a comparison between measured changes in (BMD) in a periprosthetic Femur with a cementless Bicontact stem by prospective dualenergy X-ray absorptiometry study (DEXA)and calculated changes of the (BMD) using a finite element method (FEM). As a result, it is determined that the total deviation between the two investigations is about $18 \%$.
\end{abstract}

Keywords: Stress shielding, FEM, bone remodelling, $D E X A$, periprosthetic femur

\section{Introduction}

The total hip arthroplasty (THA) is a standard treatment for many cases of severely and degeneratively changed hip joints [1]. However, the stiffness of the prosthesis is much higher than the stiffness of the bone surrounding it, thus the hip forces would be transferred more through the body of the prosthesis than the bone tissues. These changes in loading distribution lead to decrease or increase the bone mineral density (BMD). This phenomenon is called strain adaptive bone remodelling [2]. Stress shielding is one of the main factors that cause aseptic loosening of the prosthesis in the femur [3]. Several clinical studies as well as numerical investigations try to measure or estimate the bone remodelling after implanting different type of prostheses. But a comparison between them is rarely done.

Recently a DEXA study on a group of 25 patients with cementless Bicontact long stem system (AESCULAP AG, Tuttlingen, Germany) [4]. This paper represents a comparison between this study and the estimated bone remodelling of a periprosthetic femur with the same kind of prosthesis using the finite element method (FEM) $[5,6$, $, 7]$.

\section{Methods}

25 patients with cementless Bicontact system implanted in their femurs participated in the clinical study. The proximal part of the prosthesis has a rough microporous pure titanium plasmapore coating with hydroxyapatite, while the distal end of the prosthesis's stem is smooth. The patients were scanned using HOLOGIC Discovery A S/N 80600 device (Hologic Inc., Waltham, MA, USA) perioperative one week, 6 months, one year and two years postopera- tive. The hip of every patient individually is virtually divided into seven regions of interest (ROIs) (Fig. 1 (a)) in order to achieve an accurate measuring of the BMD and its changings during the study period [4].

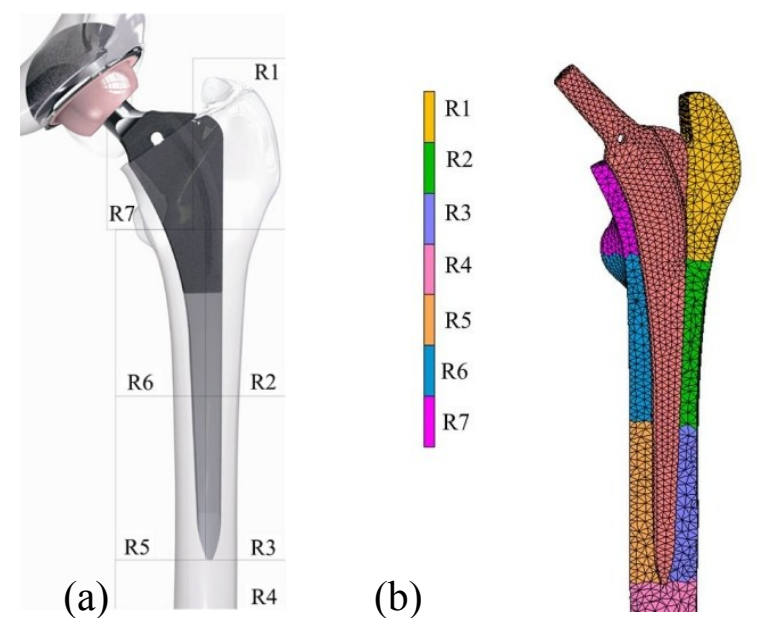

Figure 1: Region of interests on femur with the Bicontact $\mathbb{R}$ prosthesis in clinical study (a) [4] and (b) in FE-Model

The FE-model of the periprosthetic femur used at IFUM in $[5,6,7]$ is used in this comparison applying the same conditions and constraints with the same muscle forces and hip contacts but with the following restrictions:

1. The elements of the upper part of the femur are grouped in seven separated sets as stated in the clinical study in [4] (Fig. 1(b)).

2. The subroutine used in the FE calculation is modified in order to calculate the changes in bone density in each set individually.

\section{Results}

In order to be able to compare the calculated with the measured bone remodelling, the changes of the BMD from the DEXA investigation and the changes of the bone density calculated using the FE model in each ROI are given in percentage and then subtracted from each other.

The following table summarises the results of this comparison (Tab. 1):

Table 1: Calculated and measured changes in bone density (Minus means decrease).

\begin{tabular}{llll}
\hline RIO & DEXA [\%] & FEM [\%] & $\begin{array}{l}\text { Deviation } \\
{[\%]}\end{array}$ \\
\hline R1 & -12.94 & -23.5 & 10.5 \\
\hline
\end{tabular}




\begin{tabular}{llll}
\hline RIO & DEXA [\%] & FEM [\%] & $\begin{array}{l}\text { Deviation } \\
{[\%]}\end{array}$ \\
\hline R2 & -6.28 & -9.1 & 2.8 \\
R3 & -0.56 & -8.8 & 8.2 \\
R4 & +1.06 & -3.3 & 4.3 \\
R5 & -4.7 & -1.8 & 2.9 \\
R6 & +4.25 & -23.2 & 27.5 \\
R7 & -8.3 & -76.0 & 67.7 \\
mean & & & 17.7 \\
\hline
\end{tabular}

In Regions R2, R3, R4, R5 the difference between the measured and calculated bone density is around $5 \%$, while this percentage increases to $10 \%$ in $\mathrm{R} 1$. The deviation between the FE calculation and DEXA investigation is especially noticed in R7 und R6, where supposedly a strong decrease of the bone density should take place in response to the unloading of periprosthetic bone tissues expected in these regions (Fig. 2). The overall deviation in all regions is about $18 \%$, which can be considered as a good result taking into account that the stress shielding is one of many biological and mechanical factors affecting the growth of periprosthetic bone tissues.

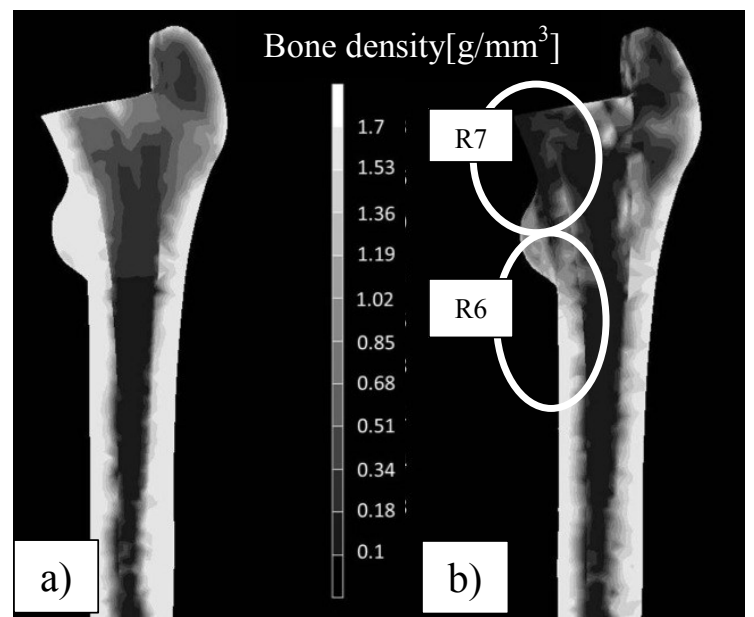

Figure 2: Bone remodelling calculated using FEM a) at the initial state and $b$ ) at the final state.

\section{Discussion}

In order to understand this difference, the physiological influences of the proximal hydroxyapatite coating and microporous structure of the prosthesis surface should be considered. These proximal properties of the Bicontact encourage the bone ingrowth in the microporous layer, which mitigates the stress concentration and thus un- as well as overloading ratio of the bone tissue [8]. The proliferation of the bone cells would be increased as another consequence of these coating properties [9]. Both effects might be reasons why the bone in R1, R6 and R7 does not lose its density as in FE-Modell expected.

In other words, the bone remodelling as a reaction of the unloading would be first activated by higher unloading ratio in regions, where bone tissue grows in the microporous prosthesis surface with hydroxyapatite coating.
In general, it can be stated that the numerical model used in $[5,6,7]$ is able to anticipate the bone remodelling with an acceptable deviation.

\section{Acknowledgement}

The study was realised in the subproject D6 of the collaborative research centre 599 "Sustainable degradable and permanent prostheses out of metallic and ceramic materials". The authors would like to thank the German Research foundation (DFG) for the financial support of the project and also the company AESCULAP AG for providing $\mathrm{CAD}$ data of the BiCONTACT ${ }^{\circledR} \mathrm{N}$ femoral component

\section{Bibliography}

[1] F. Adam, D. Kohn: Computergestützte Entwicklung eines anatomischen Hüftprothesenschaftes. Magazin Forschung, 1, 41-48, 2000

[2] D. R. Sumner, J.O. Galante: determinants of stress shielding. Clinical Orthopaedics and related Research, 274, 203-212, 1992

[3] J. Wolff: Das Gesetz der Transformation der Knochen.. Berlin, Hirschwald, 1892

[4] M. Lerch, A. Kurtz, H. Windhagen, A. Bouguecha, B.A. Behrens, P. Wefstaedt, C.M. Stukenborg-Colsman: the cementless Bicontact ${ }^{\circledR}$ stem in a prospective dualenergy X-ray absorptiometry study, International Orthopaedics (SICOT) 36:2211-2217, 2012

[5] B-A. Behrens1, I. Nolte, P. Wefstaedt, C. StukenborgColsman, A. Bouguecha: numerical investigations on the strain-adaptive bone remodeling in the periprosthetic femur: Influence of the boundary conditions BioMedical Engineering OnLine, 8:7, 2009

[6] B-A. Behrens, C.J. Wirth, H. Windhagen, I. Nolte, A. Meyer-Lindenberg, A. Bouguecha: Numerical investigations of stress shielding in total hip prostheses. Proc Inst Mech Eng H. Jul;222(5):593-600, 2008

[7] A. Bouguecha, B-A. Behrens, A. Meyer-Lindenberg, P. Wefstaedt, C. Stukenborg-Colsman, I. Nolte: Strainadaptive bone modelling: Influence of the implant material. 16th Congress of the European Society of Biomechanics, 06.-09.07. Luzern, Schweiz. 2008.

[8] L. Huanlong, S. Oppenheimer, S. Stupp, D. Dunand, L. Brinson: effects of pore morphology and bone ingrowth on mechanical properties of microporous titanium as an orthopedic implant material, Materials Transactions, Vol. 45, No. 4 pp. 1124 to 1131,2004

[9] M. Rouahi, O. Gallet, E. Champion, J. Dentzer, P. Hardouin, K. Anselme: influence of hydroxyapatite microstructure on human bone cell response, Wiley InterScience. DOI: 10.1002/jbm.a.30682, 2006 